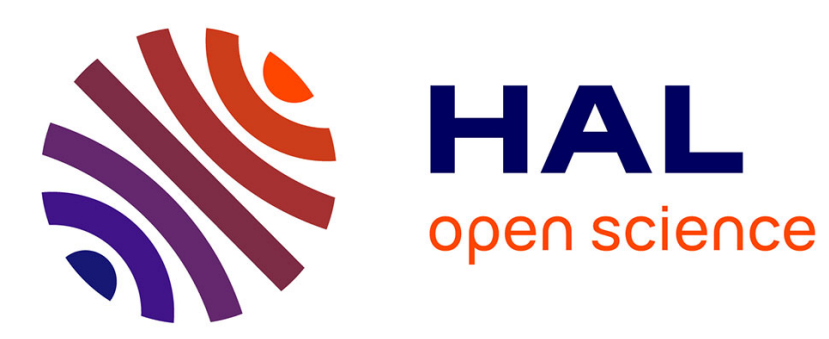

\title{
On a magnetic skin effect in eddy current problems: the magnetic potential in magnetically soft materials
}

\author{
Victor Péron, Clair Poignard
}

\section{To cite this version:}

Victor Péron, Clair Poignard. On a magnetic skin effect in eddy current problems: the magnetic potential in magnetically soft materials. Zeitschrift für Angewandte Mathematik und Physik, 2021, 10.1007/s00033-021-01596-6 . hal-02968889

\section{HAL Id: hal-02968889 \\ https://hal.inria.fr/hal-02968889}

Submitted on 16 Oct 2020

HAL is a multi-disciplinary open access archive for the deposit and dissemination of scientific research documents, whether they are published or not. The documents may come from teaching and research institutions in France or abroad, or from public or private research centers.
L'archive ouverte pluridisciplinaire HAL, est destinée au dépôt et à la diffusion de documents scientifiques de niveau recherche, publiés ou non, émanant des établissements d'enseignement et de recherche français ou étrangers, des laboratoires publics ou privés. 


\title{
ON A MAGNETIC SKIN EFFECT IN EDDY CURRENT PROBLEMS : THE MAGNETIC POTENTIAL IN MAGNETICALLY SOFT MATERIALS
}

\author{
VICTOR PÉRON AND CLAIR POIGNARD
}

\begin{abstract}
This work is concerned with the time-harmonic eddy current problem in a bidimensional setting with a high contrast of magnetic permeabilities between a conducting medium and a dielectric medium. We describe a magnetic skin effect by deriving rigorously a multiscale expansion for the magnetic potential in power series of a small parameter $\varepsilon$ which represents the inverse of the square root of a relative permeability. We make explicit the first asymptotics up to the order $\varepsilon^{3}$. As an application we obtain impedance conditions up to the fourth order of approximation for the magnetic potential. Finally we measure this skin effect with a characteristic length that depends on the scalar curvature of the boundary of the conductor.
\end{abstract}

\section{CONTENTS}

1. Introduction

2. Statement of the problem and main results 3

2.1. Uniform estimates 4

2.2. Application: Convergence of multiscale expansions 5

2.3. Impedance boundary conditions 6

2.4. A measure of the magnetic skin effect 9

3. Proofs of uniform estimates and stability results for asymptotic models 10

3.1. Proof of uniform estimates 10

3.2. Proof of stability results for asymptotic models 11

4. First terms of the multiscale expansion 12

4.1. Notations 12

4.2. First terms 12

5. Existence and regularity of the asymptotics 13

$\begin{array}{ll}\text { 5.1. Regularity results for the first terms } & 13\end{array}$

5.2. Synthesis of the multiscale expansion 14

6. Elements of derivations for the multiscale expansion and impedance conditions $\quad 15$

6.1. Expansion of the operators in power series of $\varepsilon \quad 16$

6.2. Equations for the coefficients of the magnetic potential 16

$\begin{array}{ll}\text { 6.3. First terms of the asymptotics for the magnetic potential } & 17\end{array}$

6.4. Construction of impedance conditions 18

$\begin{array}{lr}\text { Acknowledgements } & 19\end{array}$

$\begin{array}{ll}\text { References } & 20\end{array}$

Date: October 9, 2020.

Key words and phrases. Asymptotic Expansions, Impedance Conditions, Eddy Current Problems, Magnetic Potential, Ferromagnetic Material. 


\section{INTRODUCTION}

Numerical simulation of eddy current problems is crucial for the design of industrial systems. We intend to work in the context of this application to devise numerical models for the magnetic potential in (linear) magnetically soft materials. These materials are used for instance for the cores of transformers in order to reduce the energy losses associated with the reversing fields of the alternating currents. However such magnetic materials may have high magnetic permeability and a small skin depth. This raises the difficulty of applying a finite element method on a mesh that combines thin cells inside a skin depth and much larger cells outside this zone. To overcome this difficulty, it is possible to develop an asymptotic method to derive reduced models that can be solve numerically by using a coarse mesh.

To this end, we consider the derivation of an asymptotic expansion for the magnetic potential in high relative permeability $\left(\mu_{r}=\mu_{-} / \mu_{+} \gg 1\right)$. We are interested in this issue for the magnetic potential in a soft magnetic conducting body with constant permeability $\mu=\mu_{-} \gg \mu_{0}\left(\mu_{0}\right.$ is the vacuum permeability) embedded in a magnetic dielectric medium with constant permeability $\mu=\mu_{+}$, in a bi-dimensional setting. We consider the conductivity $\sigma$, as well as the angular frequency $\omega$, as given parameters.

We emphasize that the above framework will be helpful to devise $\varepsilon$-parameterizations $(\varepsilon=$ $\left.\frac{1}{\sqrt{\mu_{r}}} \ll 1\right)$ for the magnetic potential by combining the new asymptotic models with finite element solutions. We previously developed $\delta$-parameterizations ( $\delta \ll 1$ is the skin depth) for the magnetic potential in non-magnetic materials in high frequency or high conductivity (i.e. when $\omega \mu_{0} \sigma$ tends to infinity), see e.g. [10].

Roughly speaking, the aim of this paper is to provide the asymptotic expansion of the magnetic potential as the parameter $\varepsilon$ tends to zero when the surface of the conducting body is smooth. We exhibit the first terms of a singular expansion in power series of $\varepsilon$ to describe a boundary layer inside the magnetic conductor in a vicinity of its surface. In the dielectric medium $\Omega_{+}$, the magnetic potential has a regular expansion in powers of $\varepsilon$. This asymptotic expansion is validated by proving error estimates. As a by-product of this expansion, we infer high order impedance boundary conditions on the surface of the conductor.

Impedance Conditions (ICs) are usually introduced to reduce the computational domain, see for instance [11, 16, 9, 1, 8] for scattering problems from highly absorbing obstacles. The main idea consists in replacing the "exact" model inside a part of the domain by an approximate boundary condition. This idea is pertinent when the IC can be readily handled for numerical computations, for instance when this condition is local, see for instance [7, 2].

We refer the reader to $[13,8,14]$ for similar issues in the context of a non-magnetic conductor when the product $\omega \sigma$ tends to infinity (large frequency or high conductivity limit). In this case, a boundary layer phenomenon occurs near the surface of a conductor body; this is the so-called skin effect $[15,12]$. In [13] the authors validate rigorously a multiscale expansion at any order for the solution of an eddy current problem which is addressed in a bi-dimensional setting where the domain is unbounded and the solution grows logarithmically at infinity. In the work in Ref. [8] the authors address the issue of impedance conditions for the skin effect problem. They derive rigorously ICs up to the fourth order of approximation for a scalar transmission problem (Helmholtz equation) in 3D.

In this paper, we obtain a family of "Dirichlet-to-Neumann" IBCs (Section 2.3). We observe that there is a main difference between the nature of the limit problem when the product 
$\omega \mu_{0} \sigma$ tends to infinity (large frequency or high conductivity limit) [14] and the nature of the limit when the relative permeability $\mu_{r}$ tends to infinity. Indeed, the limit in the first case is simply the homogeneous Dirichlet problem for the Laplace operator set in the dielectric medium $\Omega_{+}$ (corresponding to a Perfect conductor impedance boundary condition (IBC) on the surface of the conductor) whereas the limit in the second case is the homogeneous mixed Dirichlet-Neumann problem for the Laplace operator set in $\Omega_{+}$(Problem (4.1)), corresponding to a Perfect magnetic conductor IBC on the surface of the conductor. Nonetheless, the second order IBC in high relative permeability (Eq. (2.13)) is nothing but the so-called Leontovich BC as in large frequency / conductivity [14, Eq. (A.2)] (Section 2.3.2). The third order IBC in high relative permeability is a modified Leontovich BC (Eq. (2.14)). The fourth order IBC in high relative permeability is a Ventcell condition (Eq. (2.15)). We recover the higher order IBCs in large frequency / high conductivity [14], which are of "Neumann-to-Dirichlet" nature, from the higher order IBCs in high relative permeability by truncating Taylor expansions of the formal inverses of the impedance operators (Section 2.3.2).

With this work, we observe that the asymptotic expansion for the magnetic potential in high relative permeability (Section 4) is different from the asymptotic expansion in the large frequency / high conductivity case [14]. There is also a main difference between the regularity in Sobolev spaces of the first asymptotics in high relative permeability (Section 5) and in the second case [14] since the derivate consuming processes are different. Contrary to the high conductivity case, the regular part of the expansion in high relative permeability does not consume any derivative up to the order $\varepsilon^{2}$. In particular, for given $L^{2}$ data and in smooth domains, the first four terms of the regular expansion are well-defined in Sobolev spaces based on $\mathrm{H}^{1}$ in the first case (Section 5) whereas only the first two terms are well-defined in Sobolev spaces in the second case.

The outline of the paper proceeds as follows. In Section 2, we introduce the studied problem and we present the main results. We state uniform estimates with respect to the small parameter $\varepsilon$ for solutions of the problem in Lipschitz domains. We apply this result to the convergence study of an asymptotic expansion in power series of $\varepsilon$ as $\varepsilon$ tends to zero. As a by-product of this expansion, we infer a hierarchy of impedance boundary conditions (IBCs) up to the fourth order of approximation. In Section 2.4, we measure the magnetic skin effect by introducing a characteristic length that turns out to depend on the scalar curvature of the interface. In Section 3 , we prove uniform estimates, stability and convergence results for reduced models with IBCs. In Section 4, we present the first terms of the asymptotic expansion. Then we prove existence and regularity results for the asymptotics and we present a synthesis of the multiscale expansion, Section 5. In Section 6, we give elements of derivations for the multiscale expansion and we present a formal derivation of impedance conditions.

\section{STATEMENT OF THE PROBLEM AND MAIN RESUlTS}

Let $\Omega$ be a smooth bounded domain of the plane $\mathbb{R}^{2}$ with boundary $\partial \Omega$, and $\Omega_{-} \subset \subset \Omega$ be a smooth connected subdomain of $\Omega$, with boundary $\Sigma$. We denote by $\Omega_{+}$the complementary of $\overline{\Omega_{-}}$in $\Omega$, see Fig. 1 .

The magnetic vector potential $\mathcal{A}$ reduced to a single scalar component satisfies the following problem

$$
-\operatorname{div}\left(\frac{1}{\mu} \nabla \mathcal{A}\right)+\mathrm{i} \omega \sigma \mathbb{1}_{\Omega_{-}} \mathcal{A}=J \quad \text { in } \quad \Omega .
$$




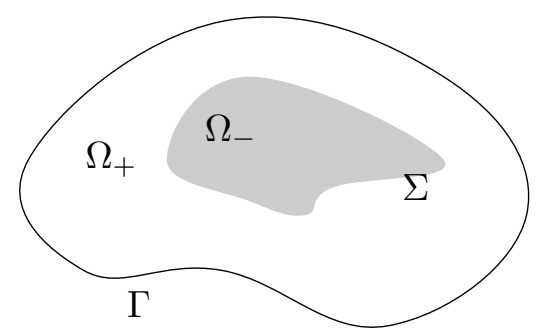

Figure 1 - The domain $\Omega$ and its subdomains $\Omega_{-}, \Omega_{+}$

Here the magnetic permeability $\mu$ is piecewise constant on $\Omega_{ \pm}$with positive real values : $\mu=$ $\mu_{ \pm}>0$ in $\Omega_{ \pm}$. In this paper we shall use the following notation for the relative magnetic permeability, which is assumed to be a large parameter

$$
\mu_{r}=\mu_{-} / \mu_{+} \gg 1 \text {. }
$$

In (2.1), the frequency $\omega>0$ and the conductivity $\sigma>0$ (in $\Omega_{-}$) are given data. For the sake of simplicity, we assume that the right-hand side $J$ (which represents a current source term) is a smooth function which has a support in $\Omega_{+}$.

Notation 2.1. We shall denote by $h^{+}\left(\right.$resp. $\left.h^{-}\right)$the restriction of any function $h$ to $\Omega_{+}$(resp. $\left.\Omega_{-}\right)$.

We introduce the small parameter $\varepsilon=\frac{1}{\sqrt{\mu_{r}}}>0$ inside the problem (2.1).

Then the magnetic vector potential $\mathcal{A}_{\varepsilon}=\left(\mathcal{A}_{\varepsilon}^{+}, \mathcal{A}_{\varepsilon}^{-}\right)$satisfies the following boundary value problem

$$
\left\{\begin{array}{lll}
-\Delta \mathcal{A}_{\varepsilon}^{+} & =\mu_{+} J & \text { in } \Omega_{+}, \\
-\varepsilon^{2} \Delta \mathcal{A}_{\varepsilon}^{-}+\mathrm{i} \omega \sigma \mu_{+} \mathcal{A}_{\varepsilon}^{-} & =0 & \text { in } \Omega_{-} \\
\mathcal{A}_{\varepsilon}^{+} & =\mathcal{A}_{\varepsilon}^{-} & \text {on } \Sigma, \\
\partial_{\mathbf{n}} \mathcal{A}_{\varepsilon}^{+} & =\varepsilon^{2} \partial_{\mathbf{n}} \mathcal{A}_{\varepsilon}^{-} & \text {on } \Sigma \\
\mathcal{A}_{\varepsilon}^{+} & =0 & \text { on } \Gamma,
\end{array}\right.
$$

where the differential operator $\Delta$ is the Laplace operator in cartesian coordinates $\mathbf{x}=(x, y) \in$ $\mathbb{R}^{2}$. As a convention, the unit normal vector $\mathbf{n}$ on the interface $\Sigma$ is inwardly oriented to $\Omega_{-}$.

Notation 2.2. We shall denote by $\mathrm{H}_{0, \Gamma}^{1}\left(\Omega_{+}\right)$the Sobolev space $\left\{u \in \mathrm{H}^{1}\left(\Omega_{+}\right) \mid u=0\right.$ on $\left.\Gamma\right\}$.

2.1. Uniform estimates. In this section we assume that the domains $\Omega_{-}$and $\Omega_{+}$are Lipschitz. We present in this part uniform a priori estimates with respect to the parameter $\varepsilon$ for the solution of the following boundary value problem

$$
\left\{\begin{array}{lll}
-\Delta \varphi^{+} & =\mu_{+} f^{+} & \text {in } \Omega_{+}, \\
-\varepsilon^{2} \Delta \varphi^{-}+\mathrm{i} \omega \sigma \mu_{+} \varphi^{-} & =f^{-} & \text {in } \Omega_{-}, \\
\varphi^{+} & =\varphi^{-} & \text {on } \Sigma, \\
\partial_{\mathbf{n}} \varphi^{+} & =\varepsilon^{2} \partial_{\mathbf{n}} \varphi^{-}+g & \text { on } \Sigma,
\end{array}\right.
$$


associated with Dirichlet external boundary conditions on $\Gamma$. The functional space suitable for its variational formulation is the Sobolev space $V=\mathrm{H}_{0}^{1}(\Omega)$. Then the variational problem writes: Find $\varphi \in V$ such that

$$
\begin{array}{r}
\forall \psi \in V, \quad \int_{\Omega_{+}} \nabla \varphi^{+} \cdot \nabla \bar{\psi}^{+} \mathrm{d} \mathbf{x}+\varepsilon^{2} \int_{\Omega_{-}} \nabla \varphi^{-} \cdot \nabla \bar{\psi}^{-} \mathrm{d} \mathbf{x}+\mathrm{i} \omega \sigma \mu_{+} \int_{\Omega_{-}} \varphi^{-} \bar{\psi}^{-} \mathrm{d} \mathbf{x}= \\
\mu_{+} \int_{\Omega} f \bar{\psi} \mathrm{d} \mathbf{x}+\langle g, \psi\rangle_{\mathrm{H}^{-\frac{1}{2}}(\Sigma), \mathrm{H}^{\frac{1}{2}}(\Sigma)} .
\end{array}
$$

Here the right-hand sides $f$ and $g$ satisfy the regularity assumption

$$
f \in \mathrm{L}^{2}(\Omega) \text { and } g \in \mathrm{H}^{-\frac{1}{2}}(\Sigma) \text {. }
$$

In this framework, there exists a unique solution $\varphi$ in $V$ to Problem (2.4) for any parameter $\varepsilon>0$.

Statement of uniform estimates. In the framework above it is possible to prove $\mathrm{H}^{1}$ a priori estimate, uniform with respect to the parameter $\varepsilon$.

Theorem 2.3. Let us assume that $\omega, \sigma, \mu_{+} \neq 0$. Then there exists $\varepsilon_{0}>0$ such that for all $\varepsilon \in\left(0, \varepsilon_{0}\right)$, the problem (2.4) with data $f$ and $g$ satisfying (2.5) has a unique solution $\varphi \in V$ which satisfies the uniform estimate

$$
\left\|\varphi^{+}\right\|_{1, \Omega_{+}}+\varepsilon\left\|\nabla \varphi^{-}\right\|_{0, \Omega_{-}}+\left\|\varphi^{-}\right\|_{0, \Omega_{-}} \leqslant C\left(\left|\mu_{+}\right|\|f\|_{0, \Omega}+\|g\|_{-\frac{1}{2}, \Sigma}\right)
$$

with a constant $C>0$, independent of $\varepsilon, f$, and $g$.

Note that these estimates are different from uniform estimates in large frequency or high conductivity [14]. We prove Theorem 2.3 in section 3.1.

2.2. Application: Convergence of multiscale expansions. In this section we assume that $\Sigma$ is a smooth curve. Then it is possible to derive a multiscale expansion for the solution $\mathcal{A}_{\varepsilon}=$ $\left(\mathcal{A}_{\varepsilon}^{+}, \mathcal{A}_{\varepsilon}^{-}\right)$of the model problem (2.3).

The solution $\mathcal{A}_{\varepsilon}$ possesses an asymptotic expansion in power series of the small parameter $\varepsilon$

$$
\begin{gathered}
\mathcal{A}_{\varepsilon}^{+}(\mathbf{x})=\mathcal{A}_{0}^{+}(\mathbf{x})+\varepsilon \mathcal{A}_{1}^{+}(\mathbf{x})+\varepsilon^{2} \mathcal{A}_{2}^{+}(\mathbf{x})+\cdots \quad \text { in } \Omega_{+}, \\
\mathcal{A}_{\varepsilon}^{-}(\mathbf{x})=\mathcal{A}_{0}^{-}(\mathbf{x} ; \varepsilon)+\varepsilon \mathcal{A}_{1}^{-}(\mathbf{x} ; \varepsilon)+\varepsilon^{2} \mathcal{A}_{2}^{-}(\mathbf{x} ; \varepsilon)+\cdots \text { in } \Omega_{-}, \\
\text {with } \mathcal{A}_{j}^{-}(\mathbf{x} ; \varepsilon)=\chi(\nu) \mathfrak{A}_{j}\left(\xi, \frac{\nu}{\varepsilon}\right) .
\end{gathered}
$$

Here $\mathbf{x} \in \mathbb{R}^{2}$ are the cartesian coordinates and $(\xi, \nu)$ is a "curvilinear coordinate system" to the curve $\Sigma$ on the domain $\Omega_{-}: \xi$ is a curvilinear abscissa on $\Sigma$ and $\nu \in\left(0, \nu_{0}\right)$ (where $\nu_{0}$ is sufficiently small, see Section 6) is the distance to the curve $\Sigma$. In Eq. (2.9), $\chi$ is a smooth cutoff function, its support is included in a tubular neighborhood of $\Sigma$, and equal to 1 in a smaller neighborhood. The first-order terms $\mathfrak{A}_{j}$ and $\mathcal{A}_{j}^{+}$are explicited in Sec. 4.2. Each term $\mathfrak{A}_{j}$ is a "profile" defined on $\Sigma \times I$ where $I=(0,+\infty)$. Furthermore, for any $j \in \mathbb{N}$

$$
\mathcal{A}_{j}^{+} \in \mathrm{H}^{1}\left(\Omega_{+}\right) \quad \text { and } \quad \mathfrak{A}_{j} \in \mathrm{H}^{1}\left(\Sigma \times \mathbb{R}^{+}\right),
$$

see Prop. 5.2 for precise results. The validation of the asymptotic expansion (2.7)-(2.8) consists in proving estimates for the remainder $r_{m ; \varepsilon}$ defined at any order $m \in \mathbb{N}^{*}$ as

$$
r_{m ; \varepsilon}=\mathcal{A}_{\varepsilon}-\sum_{n=0}^{m} \varepsilon^{n} \mathcal{A}_{n} \quad \text { in } \Omega .
$$


It is possible to prove estimates at any order $m \in \mathbb{N}^{*}$ for the remainder $r_{m ; \varepsilon}$. We can prove the following convergence result as a consequence of Th. 2.3.

Theorem 2.4. We assume that $J$ is a smooth data and the interface $\Sigma$ is a smooth curve. There exists $\varepsilon_{0}>0$ such that for all $m \in \mathbb{N}$ there exists a constant $C_{m}>0$ independent of $\varepsilon$ such that for all $\varepsilon \in\left(0, \varepsilon_{0}\right)$ the remainder $r_{m ; \varepsilon}$ satisfies the optimal estimate

$$
\left\|r_{m ; \varepsilon}^{+}\right\|_{1, \Omega_{+}}+\varepsilon\left\|\nabla r_{m ; \varepsilon}^{-}\right\|_{0, \Omega_{-}}+\left\|r_{m ; \varepsilon}^{-}\right\|_{0, \Omega_{-}} \leqslant C_{m} \varepsilon^{m+1} .
$$

Proof. This proof is done by an evaluation of the right-hand side when the operator $-\operatorname{div}\left(\frac{1}{\mu} \nabla\right)+$ $\mathrm{i} \omega \sigma \mathbb{1}_{\Omega_{-}}$is applied to the remainder $r_{m ; \varepsilon}$. By construction of the expansion (2.7)-(2.8) we obtain (setting $\delta_{0}=\frac{1}{\sqrt{\omega \sigma \mu_{+}}}$)

$$
\left\{\begin{array}{lll}
-\Delta r_{m ; \varepsilon}^{+} & =0 & \text { in } \Omega_{+}, \\
-\varepsilon^{2} \Delta r_{m ; \varepsilon}^{-}+\mathrm{i} \delta_{0}^{-2} r_{m ; \varepsilon}^{-} & =f_{m ; \varepsilon}^{-} & \text {in } \Omega_{-}, \\
r_{m ; \varepsilon}^{+} & =r_{m ; \varepsilon}^{-} & \text {on } \Sigma, \\
\partial_{\mathbf{n}} r_{m ; \varepsilon}^{+} & =\varepsilon^{2} \partial_{\mathbf{n}} r_{m ; \varepsilon}^{-}+\varepsilon^{m+1} \partial_{\mathbf{n}} \mathcal{A}_{m+1}^{+} & \text {on } \Sigma, \\
r_{m ; \varepsilon}^{+} & =0 & \text { on } \Gamma .
\end{array}\right.
$$

Since $J$ is smooth, the right-hand side $f_{m ; \varepsilon}^{-}$belongs to $\mathrm{L}^{2}\left(\Omega_{-}\right)$and it is roughly of the order $\varepsilon^{m+1}$; the right-hand side $\partial_{\mathbf{n}} \mathcal{A}_{m+1}^{+}$belongs to $\mathrm{H}^{-\frac{1}{2}}(\Sigma)$ and it is independent of $\varepsilon$. Then using estimates (2.6) we infer the error estimate (2.10).

2.3. Impedance boundary conditions. As a by-product of the multiscale expansion it is possible to derive high-order impedance boundary conditions (ICs) set on the interface $\Sigma$ for the solution of the problem (2.3). Elements of formal derivations are given in Section 6.4.

2.3.1. Statement of impedance boundary conditions. This section presents a hierarchy of ICs up to the fourth order of approximation with respect to the parameter $\varepsilon$, See Thm. 2.7 (§2.3.4) for precise estimates.

Order 1: Perfect magnetic conductor b.c.

$$
\partial_{\mathbf{n}} \mathcal{A}_{0}=0 \quad \text { on } \quad \Sigma
$$

Order 2: Leontovich b.c.

$$
\partial_{\mathbf{n}} \mathcal{A}_{1}^{\varepsilon}+\varepsilon \lambda_{0} \mathcal{A}_{1}^{\varepsilon}=0 \quad \text { on } \quad \Sigma
$$

where $\lambda_{0}=\sqrt{\omega \sigma \mu_{+}} \mathrm{e}^{\frac{\mathrm{i} \pi}{4}}$.

Order 3: Modified Leontovich b.c.

$$
\partial_{\mathbf{n}} \mathcal{A}_{2}^{\varepsilon}+\varepsilon\left(\lambda_{0}-\frac{k(\xi)}{2} \varepsilon\right) \mathcal{A}_{2}^{\varepsilon}=0 \quad \text { on } \quad \Sigma
$$

Here $k$ denotes the scalar curvature of the curve $\Sigma$ (see Not. 4.1). 
Order 4: Ventcell b.c.

$$
\partial_{\mathbf{n}} \mathcal{A}_{3}^{\varepsilon}+\varepsilon\left(\lambda_{0}-\frac{k(\xi)}{2} \varepsilon-\frac{\varepsilon^{2}}{2 \lambda_{0}}\left\{\partial_{\xi}^{2}+\frac{k(\xi)^{2}}{4} \mathbb{I}\right\}\right) \mathcal{A}_{3}^{\varepsilon}=0 \quad \text { on } \quad \Sigma
$$

2.3.2. Comparison with the large frequency / high conductivity case [14]. The Leontovich b.c. (2.13) writes

$$
\partial_{\mathbf{n}} \mathcal{A}_{1}^{\varepsilon}+\sqrt{\frac{\omega \sigma}{\mu_{-}}} \mu_{+} \mathrm{e}^{\frac{\mathrm{i} \pi}{4}} \mathcal{A}_{1}^{\varepsilon}=0 \quad \text { on } \quad \Sigma .
$$

This IC is nothing but the second order IC for the large frequency / high conductivity case [14, Eq. (A.2)].

The modified Leontovich b.c. (2.14) writes

$$
\partial_{\mathbf{n}} \mathcal{A}_{2}^{\varepsilon}+\sqrt{\frac{\omega \sigma}{\mu_{-}}} \mu_{+} \mathrm{e}^{\frac{\mathrm{i} \pi}{4}}\left(1-\frac{1}{\sqrt{\omega \sigma \mu_{-}}} \frac{k(\xi)}{2} \mathrm{e}^{-\frac{\mathrm{i} \pi}{4}}\right) \mathcal{A}_{2}^{\varepsilon}=0 \quad \text { on } \quad \Sigma .
$$

From this IC, we recover the third order IC in the large frequency / high conductivity case [14, Eq. (A.3)] by performing a first order truncated Taylor expansion of the formal operator

$$
\begin{aligned}
& \left(\mathbb{I}-\frac{1}{\sqrt{\omega \sigma \mu_{-}}} \frac{k(\xi)}{2} \mathrm{e}^{-\frac{\mathrm{i} \pi}{4}} \mathbb{I}\right)^{-1} \text { as the parameter } \frac{1}{\sqrt{\omega \sigma}} \text { tends to zero : } \\
& \mathcal{A}+\sqrt{\frac{\mu_{-}}{\omega \sigma}} \frac{1}{\mu_{+}} \mathrm{e}^{-\frac{\mathrm{i} \pi}{4}}\left(1+\frac{1}{\sqrt{\omega \sigma \mu_{-}}} \frac{k(\xi)}{2} \mathrm{e}^{-\frac{\mathrm{i} \pi}{4}}\right) \partial_{\mathbf{n}} \mathcal{A}=0 \quad \text { on } \quad \Sigma .
\end{aligned}
$$

This Neumann-to-Dirichlet IC can also be obtained from the modified Leontovich b.c. (2.14) by truncating a first order Taylor expansion of the formal operator $\left(\lambda_{0} \mathbb{I}-\frac{k(\xi)}{2} \varepsilon \mathbb{I}\right)^{-1}$ as $\varepsilon$ tends to zero.

The Ventcell b.c. writes

$\partial_{\mathbf{n}} \mathcal{A}_{3}^{\varepsilon}+\sqrt{\frac{\omega \sigma}{\mu_{-}}} \mu_{+} \mathrm{e}^{\frac{\mathrm{i} \pi}{4}}\left(1-\frac{1}{\sqrt{\omega \sigma \mu_{-}}} \frac{k(\xi)}{2} \mathrm{e}^{-\frac{\mathrm{i} \pi}{4}}+\frac{1}{\omega \sigma \mu_{-}} \frac{\mathrm{i}}{2}\left\{\partial_{\xi}^{2}+\frac{k(\xi)^{2}}{4} \mathbb{I}\right\}\right) \mathcal{A}_{3}^{\varepsilon}=0 . \quad$ on $\quad \Sigma$

From this IC, we recover the fourth order IC in the large frequency / high conductivity case [14, Eq. (A.4)] by performing a second order truncated Taylor expansion of the formal operator

$$
\left(\mathbb{I}-\frac{1}{\sqrt{\omega \sigma \mu_{-}}} \frac{k(\xi)}{2} \mathrm{e}^{-\frac{\mathrm{i} \pi}{4}} \mathbb{I}+\frac{1}{\omega \sigma \mu_{-}} \frac{\mathrm{i}}{2}\left\{\partial_{\xi}^{2}+\frac{k(\xi)^{2}}{4} \mathbb{I}\right\}\right)^{-1} \text { as the parameter } \frac{1}{\sqrt{\omega \sigma}} \text { tends to }
$$

$\mathcal{A}+\sqrt{\frac{\mu_{-}}{\omega \sigma}} \frac{1}{\mu_{+}} \mathrm{e}^{-\frac{\mathrm{i} \pi}{4}}\left(1+\frac{1}{\sqrt{\omega \sigma \mu_{-}}} \frac{k(\xi)}{2} \mathrm{e}^{-\frac{\mathrm{i} \pi}{4}}-\frac{1}{\omega \sigma \mu_{-}} \frac{\mathrm{i}}{2}\left\{\partial_{\xi}^{2}+\frac{k(\xi)^{2}}{4} \mathbb{I}\right\}\right) \partial_{\mathbf{n}} \mathcal{A}=0 \quad$ on $\quad \Sigma$.

This Neumann-to-Dirichlet IC can also be obtained from the Ventcell b.c. (2.15) by truncating a second order Taylor expansion of the formal operator $\left(\lambda_{0} \mathbb{I}-\frac{k(\xi)}{2} \varepsilon \mathbb{I}-\frac{\varepsilon^{2}}{2 \lambda_{0}}\left\{\partial_{\xi}^{2}+\frac{k(\xi)^{2}}{4} \mathbb{I}\right\}\right)^{-1}$ as $\varepsilon$ tends to zero.

2.3.3. Stability analysis. In the framework above, a simpler problem than the problem (2.3) writes

$$
\begin{cases}-\Delta \mathcal{A}_{k}^{\varepsilon} & =f \text { in } \Omega_{+} \\ \mathrm{N}_{k, \varepsilon}\left(\mathcal{A}_{k}^{\varepsilon}\right)+\partial_{\mathbf{n}} \mathcal{A}_{k}^{\varepsilon} & =0 \text { on } \Sigma \\ \mathcal{A}_{k}^{\varepsilon} & =0 \text { on } \Gamma\end{cases}
$$


(with $f=\mu_{+} J$ ) where $\mathrm{N}_{k, \varepsilon}$ is a Dirichlet-to-Neumann operator which is given explicitly by (2.12)-(2.13)-(2.14)-(2.15) when $k=0,1,2,3$, respectively

$$
\begin{gathered}
\mathrm{N}_{0}=0 \\
\mathrm{~N}_{1, \varepsilon}=\lambda_{0} \varepsilon \mathbb{I}, \\
\mathrm{N}_{2, \varepsilon}=\varepsilon\left(\lambda_{0}-\frac{k(\xi)}{2} \varepsilon\right) \mathbb{I}, \\
\mathrm{N}_{3, \varepsilon}=\varepsilon\left(\left(\lambda_{0}-\frac{k(\xi)}{2} \varepsilon\right) \mathbb{I}-\frac{\varepsilon^{2}}{2 \lambda_{0}}\left\{\partial_{\xi}^{2}+\frac{k(\xi)^{2}}{4} \mathbb{I}\right\}\right) .
\end{gathered}
$$

We can prove a stability result for this problem in the following functional framework which is defined as $\mathrm{V}_{k}=\mathrm{H}_{0, \Gamma}^{1}\left(\Omega_{+}\right)$(Not. 2.2) when $k=0,1,2$, and $\mathrm{V}_{k}=\left\{\varphi \in \mathrm{H}_{0, \Gamma}^{1}\left(\Omega_{+}\right) \mid \nabla_{\Sigma} \varphi \in\right.$ $\left.\mathrm{L}^{2}(\Sigma)\right\}$ when $k=3$.

Theorem 2.5. For all $k \in\{0,1,2,3\}$ there are constants $\varepsilon_{k}, C_{k}>0$ such that for all $\varepsilon \in\left(0, \varepsilon_{k}\right)$, the problem (2.16) with a data $f \in \mathrm{L}^{2}\left(\Omega_{+}\right)$has a unique solution $\mathcal{A}_{k}^{\varepsilon} \in \mathrm{V}_{k}$ which satisfies the uniform estimates:

$$
\begin{gathered}
\left\|\mathcal{A}_{k}^{\varepsilon}\right\|_{1, \Omega_{+}} \leqslant C_{k}\|f\|_{0, \Omega_{+}} \quad \text { for all } \quad k \in\{0,1,2\}, \\
\left\|\mathcal{A}_{3}^{\varepsilon}\right\|_{1, \Omega_{+}}+\varepsilon^{\frac{3}{2}}\left\|\nabla_{\Sigma} \mathcal{A}_{3}^{\varepsilon}\right\|_{0, \Sigma} \leqslant C_{k}\|f\|_{0, \Omega_{+}} .
\end{gathered}
$$

Remark 2.1. Estimate (2.18a) holds for all $\varepsilon>0$ when $k=0$, i.e. $\varepsilon_{0}=+\infty$. Estimate (2.18a) holds for all $\varepsilon \in\left(0, \sqrt{2}\left\|_{\frac{1}{k(\xi)}}\right\|_{\infty, \Sigma}\right)$ when $k=1$.

The key for the proof of this result is the following Lemma, that is uniform $\mathrm{L}^{2}$-estimates for any solution of problem (2.16)

Lemma 2.6. For all $k \in\{0,1,2,3\}$ there are constants $\varepsilon_{k}, C_{k}>0$ such that for all $\varepsilon \in\left(0, \varepsilon_{k}\right)$, any solution $\mathcal{A}_{k}^{\varepsilon} \in \mathrm{V}_{k}$ of problem (2.16) with a data $f \in \mathrm{L}^{2}\left(\Omega_{+}\right)$satisfies the uniform estimate

$$
\left\|\mathcal{A}_{k}^{\varepsilon}\right\|_{0, \Omega_{+}} \leqslant C_{k}\|f\|_{0, \Omega_{+}} .
$$

This Lemma is proved in Section 3.2. As a consequence of this Lemma, any solution of the problem (2.16) satisfies uniform $\mathrm{H}^{1}$-estimates (2.18a), (2.18b), respectively when $k=0,1,2,3$. Then, the proof of the Thm. 2.5 is obtained as a consequence of the Fredholm alternative since problem (2.16) is Fredholm of index zero.

2.3.4. Convergence result. We remind that $\mathcal{A}_{\varepsilon}$ is the solution of the problem (2.3). As a consequence of Thm. 2.4 and Thm. 2.5, we can deduce the following convergence result for the solution $\mathcal{A}_{k}^{\varepsilon}$ of the asymptotic model (2.16)

Theorem 2.7. We assume that $J$ is a smooth data and the interface $\Sigma$ is a smooth curve. For all $k \in\{0,1,2,3\}$ there are constants $\varepsilon_{k}, C_{k}>0$ such that for all $\varepsilon \in\left(0, \varepsilon_{k}\right), \mathcal{A}_{k}^{\varepsilon} \in \mathrm{V}_{k}$ satisfies the uniform error estimates

$$
\left\|\mathcal{A}_{\varepsilon}-\mathcal{A}_{k}^{\varepsilon}\right\|_{1, \Omega_{+}} \leqslant C_{k} \varepsilon^{k+1}
$$


2.4. A measure of the magnetic skin effect. As another application of the multiscale expansion, we make the link in this section between the parameter $\varepsilon$ and the "pointwise energy" of the solution of the problem (2.3) across the boundary layer. We measure this boundary layer phenomenon by introducing a characteristic length that turns out to depend on the scalar curvature (see Not. 4.1) of the boundary of the domain $\Omega_{-}$. An asymptotic expansion when $\varepsilon \rightarrow 0$ for this function shows the influence of the geometry of the interface : the characteristic length is larger for small values of $\varepsilon$ when the scalar curvature of the interface $\Sigma$ is larger. We refer also to the works $[6,3,4]$ where the authors measure similarly boundary layer phenomena (in 3D) by introducing suitable "skin depth" functions.

Introduction of a characteristic length. For a data $J$ of the problem (2.3), let us define $\mathcal{A}_{\varepsilon}(\xi, \nu)=$ $\mathcal{A}_{\varepsilon}^{-}(\mathbf{x}), \xi \in \Sigma, 0 \leqslant \nu<\nu_{0}$ for $\nu_{0}$ small enough.

Definition 2.8. Let $J$ be a smooth data of problem (2.3) such that for all $\xi \in \Sigma, \mathcal{A}_{\varepsilon}(\xi ; 0) \neq 0$. The characteristic length is the length $\mathcal{L}(\varepsilon ; \xi)$ defined on $\Sigma$ and taking the smallest value such that

$$
\left|\mathcal{A}_{\varepsilon}(\xi ; \mathcal{L}(\varepsilon ; \xi))\right|=\left|\mathcal{A}_{\varepsilon}(\xi ; 0)\right| \mathrm{e}^{-1}
$$

Thus this length $\mathcal{L}(\varepsilon ; \xi)$ is the distance from the interface where $\left|\mathcal{A}_{\varepsilon}\right|$ has decreased of a fixed rate e. It depends on $\varepsilon$, of each point $\xi \in \Sigma$ and a priori on the data $J$. However it is possible to exhibit the asymptotic behavior of the characteristic length $\mathcal{L}$ for small values of $\varepsilon$ independently of both $\xi$ and the data $J$.

Asymptotic behavior of the characteristic length function when $\varepsilon \rightarrow 0$.

Theorem 2.9. Let $J$ be a smooth data of problem (2.3). We assume that $\mathcal{A}_{0}^{-}(\mathbf{X}(\xi)) \neq 0$ on $\Sigma$. Then characteristic length has the following behavior for small values of $\varepsilon$

$$
\mathcal{L}(\varepsilon ; \xi) \approx \sqrt{\frac{2}{\omega \sigma \mu_{-}}}\left(1+\frac{k(\xi)}{2} \sqrt{\frac{2}{\omega \sigma \mu_{-}}}+\mathcal{O}\left(\frac{\mu_{+}}{\mu_{-}}\right)\right), \quad \varepsilon=\sqrt{\frac{\mu_{+}}{\mu_{-}}} \rightarrow 0 .
$$

Remark 2.2. This result is comparable to the skin effect in 2D. The truncated expansion formed by the two first terms in (2.22) coincides with the asymptotic behavior in high conductivity of the "skin depth" function introduced in $[6,3]$ when the permeability $\mu_{-}$is the vacuum permeability.

Proof. The proof of this theorem is based on the formula

$$
\left|\mathcal{A}_{\varepsilon}(\xi ; \nu)\right|=\left|\mathcal{A}_{\varepsilon}(\xi ; 0)\right| \mathrm{m}(\xi ; \nu) \mathrm{e}^{-\nu \frac{\Re \lambda_{0}}{\varepsilon}}
$$

with

$$
\mathrm{m}(\xi ; \nu)=1+\frac{k(\xi)}{2 \Re \lambda_{0}} \nu+\mathcal{O}\left((\varepsilon+\nu)^{2}\right) .
$$

This formula (2.23) is a consequence of equations (4.2) and (4.4). According to Definition 2.8, the characteristic length $\mathcal{L}(\varepsilon ;$.$) satisfies$

$$
\left|\mathcal{A}_{\varepsilon}(\xi ; \mathcal{L}(\varepsilon ; \xi))\right|=\left|\mathcal{A}_{\varepsilon}(\xi ; 0)\right| \mathrm{e}^{-1} .
$$

Hence,

$$
\mathrm{m}(\xi ; \mathcal{L}(\varepsilon ; \xi)) \mathrm{e}^{-\mathcal{L}(\varepsilon ; \xi) \Re \lambda_{0} / \varepsilon}=\mathrm{e}^{-1}
$$


Performing an asymptotic expansion when $\varepsilon$ tends to zero, we infer the asymptotic behavior of the characteristic length (2.22)

$$
\mathcal{L}(\varepsilon ; \xi) \approx \varepsilon\left(\Re \lambda_{0}\right)^{-1}\left(1+\frac{k(\xi)}{2 \Re \lambda_{0}} \varepsilon+\mathcal{O}\left(\varepsilon^{2}\right)\right), \quad \varepsilon \rightarrow 0 .
$$

\section{PROOFS OF UNIFORM ESTIMATES AND STABILITY RESULTS FOR ASYMPTOTIC MODELS}

3.1. Proof of uniform estimates. The proof of Theorem 2.3 is based on the following Lemma, that is uniform $\mathrm{L}^{2}$ estimates.

Lemma 3.1. There exists $\varepsilon_{0}>0$ such that for all $\varepsilon \in\left(0, \varepsilon_{0}\right)$, any solution $\varphi \in V$ of problem (2.4), with data $f$ and $g$ satisfying (2.5), satisfies the uniform estimate

$$
\|\varphi\|_{0, \Omega} \leqslant C\left(\|f\|_{0, \Omega}+\|g\|_{-\frac{1}{2}, \Sigma}\right)
$$

with a constant $C>0$, independent of $\varepsilon, f$, and $g$.

The proof of this result involves a compactness argument. Using estimates (3.1) we can deduce estimates (2.6) and Thm. 2.3 is obtained as a consequence of the Fredholm alternative since the problem (2.4) is Fredholm of index zero.

3.1.1. Proof of Lemma 3.1 : Uniform $\mathrm{L}^{2}$ estimates for $\varphi_{\varepsilon}$. Without loss of generality we can restrict our consideration by proving this lemma when $g=0$ (since it is still possible to absorb the $\mathrm{H}^{\frac{1}{2}}$ norm of $\varphi$ on $\Sigma$ with the $\mathrm{H}^{1}$ semi-norm of $\varphi^{+}$on $\Omega_{+}$from the right-hand side to the left-hand side when $g \neq 0$ ). We adopt a proof by contradiction : We assume that there exists a sequence $\left(\varphi_{m}\right) \in V, m \in \mathbb{N}$, of solutions of problem (2.4) associated with a parameter $\varepsilon_{m}$ and a right-hand side $f_{m} \in \mathrm{L}^{2}(\Omega)$ :

$$
\begin{array}{r}
\forall \psi \in V, \quad \int_{\Omega_{+}} \nabla \varphi_{m}^{+} \cdot \nabla \bar{\psi}^{+} \mathrm{d} \mathbf{x}+\varepsilon_{m}^{2} \int_{\Omega_{-}} \nabla \varphi_{m}^{-} \cdot \nabla \bar{\psi}^{-} \mathrm{d} \mathbf{x}+\mathrm{i} \omega \sigma \mu_{+} \int_{\Omega_{-}} \varphi_{m}^{-} \bar{\psi}^{-} \mathrm{d} \mathbf{x}= \\
\mu_{+} \int_{\Omega} f_{m} \bar{\psi} \mathrm{d} \mathbf{x}
\end{array}
$$

satisfying the following conditions

$$
\begin{array}{cl}
\varepsilon_{m} \rightarrow 0 & \text { as } m \rightarrow \infty, \\
\left\|\varphi_{m}\right\|_{0, \Omega}=1 & \text { for all } m \in \mathbb{N}, \\
\left\|f_{m}\right\|_{0, \Omega} \rightarrow 0 & \text { as } m \rightarrow \infty .
\end{array}
$$

Choosing tests functions $\psi=\varphi_{m}$ in (3.2) and taking the real part, we obtain the following convergence results on the gradients :

(i) the sequence $\left\{\nabla \varphi_{m}^{+}\right\}$converges to 0 in $\mathrm{L}^{2}\left(\Omega_{+}\right)$.

Then taking the imaginary part, we obtain with the help of conditions (3.3b)-(3.3c) the following convergence result :

(ii) the sequence $\left\{\varphi_{m}^{-}\right\}$converges to 0 in $\mathrm{L}^{2}\left(\Omega_{-}\right)$.

As a consequence of (3.3b) and $(i)$, the sequence $\left\{\varphi_{m}^{+}\right\}$is bounded in $\mathrm{H}^{1}\left(\Omega_{+}\right)$. 
Limit of the sequence and conclusion. The domain $\Omega_{+}$being bounded, the embedding of $\mathrm{H}^{1}\left(\Omega_{+}\right)$ in $\mathrm{L}^{2}\left(\Omega_{+}\right)$is compact (Rellich Lemma). Hence we can extract a subsequence of $\left\{\varphi_{m}^{+}\right\}$(still denoted by $\left\{\varphi_{m}^{+}\right\}$) which is strongly converging in $\mathrm{L}^{2}\left(\Omega_{+}\right)$and weakly converging in $\mathrm{H}^{1}\left(\Omega_{+}\right)$

$$
\left\{\begin{array}{lll}
\nabla \varphi_{m}^{+} \rightarrow \nabla \varphi^{+} & \text {in } & \mathrm{L}^{2}\left(\Omega_{+}\right) \\
\varphi_{m}^{+} \rightarrow \varphi^{+} & \text {in } & \mathrm{L}^{2}\left(\Omega_{+}\right)
\end{array}\right.
$$

and (i) implies that this sequence is strongly converging in $\mathrm{H}^{1}\left(\Omega_{+}\right)$and $\nabla \varphi^{+}=0$ in $\Omega_{+}$.

A consequence of the strong convergence in $\mathrm{L}^{2}(\Omega)$ and (3.3b) is that $\left\|\varphi^{+}\right\|_{0, \Omega_{+}}=1$. Since $\nabla \varphi^{+}=0$ in $\Omega_{+}$and $\varphi^{+}=0$ on $\partial \Omega$, we infer that $\varphi^{+}=0$ in $\Omega_{+}$which contradicts $\left\|\varphi^{+}\right\|_{0, \Omega_{+}}=$ 1 and finally proves estimate (3.1).

3.2. Proof of stability results for asymptotic models. We focus on the proof of Lemma 2.6 for $k=3$ since the proof is simpler when $k=0,1,2$. We present a proof which is based on a compactness argument. To prepare for this proof, we introduce the variational formulation for $\mathrm{u}=\mathcal{A}_{3}^{\varepsilon}$. If $\mathrm{u} \in \mathrm{V}_{3}$ is a solution of (2.16), then it satisfies for all $\mathrm{v} \in \mathrm{V}_{3}$

$$
\int_{\Omega_{+}} \nabla \mathbf{u} \cdot \nabla \overline{\mathrm{v}} \mathrm{d} \mathbf{x}+\varepsilon \int_{\Sigma}\left\{\left(\lambda_{0}-\frac{k(\xi)}{2} \varepsilon-\frac{k(\xi)^{2}}{8 \lambda_{0}} \varepsilon^{2}\right) \mathbf{u} \overline{\mathbf{v}}+\frac{\varepsilon^{2}}{2 \lambda_{0}} \partial_{\xi} \mathrm{u} \partial_{\xi} \overline{\mathrm{v}}\right\} \mathrm{d} \xi=\int_{\Omega_{+}} f \overline{\mathrm{v}} \mathrm{d} \mathbf{x} .
$$

Reductio ad absurdum: We assume that there is a sequence $\left\{\mathrm{u}_{m}\right\} \in \mathrm{V}_{3}, m \in \mathbb{N}$, of solutions of the problem (2.16) (when $k=3$ ) associated with a parameter $\varepsilon_{m}$ and a right-hand side $f_{m} \in$ $\mathrm{L}^{2}\left(\Omega_{+}\right)$

$$
\begin{aligned}
& -\Delta \mathrm{u}_{m}=f_{m} \quad \text { in } \quad \Omega_{+}, \\
& \partial_{\mathbf{n}} \mathbf{u}_{m}+\mathcal{J}_{m} \mathbf{u}_{m}-\frac{\varepsilon_{m}^{3}}{2 \lambda_{0}} \Delta_{\Sigma} \mathbf{u}_{m}=0 \quad \text { on } \quad \Sigma, \\
& \mathrm{u}_{m}=0 \quad \text { on } \quad \partial \Omega
\end{aligned}
$$

satisfying the following conditions

$$
\begin{array}{cl}
\varepsilon_{m} \rightarrow 0 & \text { as } m \rightarrow \infty, \\
\left\|\mathbf{u}_{m}\right\|_{0, \Omega_{+}}=1 & \text { for all } m \in \mathbb{N}, \\
\left\|f_{m}\right\|_{0, \Omega_{+}} \rightarrow 0 & \text { as } m \rightarrow \infty .
\end{array}
$$

In (3.6b), $\mathcal{J}_{m}=\varepsilon_{m}\left(\lambda_{0}-\frac{k(\xi)}{2} \varepsilon_{m}-\frac{k(\xi)^{2}}{8 \lambda_{0}} \varepsilon_{m}^{2}\right)$ is a scalar function defined on $\Sigma$.

Estimates of the sequence $\left\{\mathrm{u}_{m}\right\}$. We first prove that the sequence $\left\{\mathrm{u}_{m}\right\}$ is bounded in $\mathrm{H}^{1}\left(\Omega_{+}\right)$. We particularize the weak formulation (3.5) for the sequence $\left\{u_{m}\right\}$ and choosing $v=u_{m}$ we obtain

$$
\left\|\nabla \mathbf{u}_{m}\right\|_{0, \Omega_{+}}^{2}+\int_{\Sigma}\left\{\mathcal{J}_{m}\left|\mathbf{u}_{m}\right|^{2}+\frac{\varepsilon_{m}^{3}}{2 \lambda_{0}}\left|\partial_{\xi} \mathbf{u}_{m}\right|^{2}\right\} \mathrm{d} \xi=\int_{\Omega_{+}} f \overline{\mathbf{u}_{m}} \mathrm{~d} \mathbf{x} .
$$

Then taking the real part, we obtain with the help of condition (3.7a)-(3.7b)-(3.7c) the convergence result

$$
\nabla \mathrm{u}_{m} \longrightarrow 0 \quad \text { in } \quad \mathrm{L}^{2}\left(\Omega_{+}\right)
$$


since $\mathcal{J}_{m} \rightarrow 0$. We infer that the sequence $\left\{\mathrm{u}_{m}\right\}$ is bounded in $\mathrm{H}^{1}\left(\Omega_{+}\right)$

$$
\left\|\mathrm{u}_{m}\right\|_{1, \Omega_{+}} \leqslant C \text {. }
$$

Limit of the sequence and conclusion. The domain $\Omega_{+}$being bounded, the embedding of $\mathrm{H}^{1}\left(\Omega_{+}\right)$ in $\mathrm{L}^{2}\left(\Omega_{+}\right)$is compact (Rellich Lemma). As a consequence of (3.10), using the Rellich Lemma we can extract a subsequence of $\left\{\mathbf{u}_{m}\right\}$ (still denoted by $\left\{\mathbf{u}_{m}\right\}$ ) which is weakly converging in $\mathrm{H}^{1}\left(\Omega_{+}\right)$and strongly converging in $\mathrm{L}^{2}\left(\Omega_{+}\right)$. We deduce that there is $u \in \mathrm{L}^{2}\left(\Omega_{-}\right)$such that

$$
\begin{gathered}
\mathrm{u}_{m} \rightarrow \mathbf{u} \quad \text { in } \quad \mathrm{L}^{2}\left(\Omega_{+}\right) \\
\nabla \mathrm{u}_{m} \rightarrow \nabla \mathbf{u} \quad \text { in } \quad \mathrm{L}^{2}\left(\Omega_{+}\right) .
\end{gathered}
$$

A consequence of the strong convergence result in $\mathrm{L}^{2}\left(\Omega_{+}\right)$(3.11a) and (3.7b) is that $\|\mathrm{u}\|_{0, \Omega_{+}}=$ 1. As a consequence of (3.11b)-(3.9) we deduce that $\nabla u=0$ in $\Omega_{+}$. Since $u=0$ on $\Gamma$ we infer $\mathrm{u}=0$ in $\Omega_{+}$which contradicts $\|\mathrm{u}\|_{0, \Omega_{+}}=1$ and ends the proof.

\section{First terms OF THE MULTISCALE EXPANSION}

In this section we assume that $\Sigma$ is a smooth curve. Hereafter, we present the construction of the first profiles $\mathfrak{A}_{j}$ and of the first terms $\mathcal{A}_{j}^{+}$for the multiscale expansion (2.7)-(2.8).

\subsection{Notations.}

Notation 4.1. We denote by $\mathbf{X}(\xi) \in \Sigma$ an arc-length parametrization of $\Sigma$. Here $\mathbf{X}$ is a smooth function from $\mathbb{T}_{L}:=\mathbb{R} / L \mathbb{Z}$ into $\mathbb{R}^{2}$ and $L$ is the length of the curve $\Sigma$. The scalar curvature $k(\xi)$ at the point $\mathbf{X}(\xi) \in \Sigma$ is well-defined by

$$
\frac{d \mathbf{n}}{d \xi}=-k(\xi) \frac{d \mathbf{X}}{d \xi}
$$

We remind that $\mathbf{n}(\xi)$ denotes the unit normal at $\mathbf{X}(\xi) \in \Sigma$ which is inwardly oriented to $\Omega_{-}$.

4.2. First terms. In this section, we present the construction of the asymptotics $\left(\mathcal{A}_{j}^{+}, \mathfrak{A}_{j}\right)$ when $j=0,1,2,3$. Elements of formal derivations are given in Section 6. The first term $\mathcal{A}_{0}^{+}$solves the problem

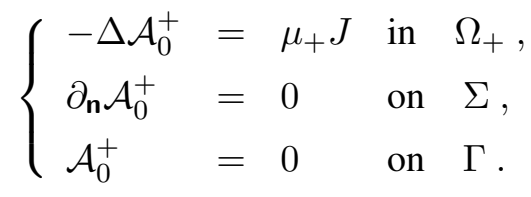

Then the first profile $\mathfrak{A}_{0}$ is

$$
\mathfrak{A}_{0}(\xi, \Upsilon)=\mathcal{A}_{0}^{+}(\mathbf{X}(\xi)) \mathrm{e}^{-\lambda_{0} \Upsilon}
$$

where $\lambda_{0}=\sqrt{\frac{\omega \sigma \mu_{+}}{2}}(1+\mathrm{i})$.

The next term which is determined in the asymptotics is $\mathcal{A}_{1}^{+}$which solves

$$
\begin{cases}\Delta \mathcal{A}_{1}^{+}=0 & \text { in } \Omega_{+}, \\ \partial_{\mathbf{n}} \mathcal{A}_{1}^{+}=-\lambda_{0} \mathcal{A}_{0}^{+} & \text {on } \Sigma, \\ \mathcal{A}_{1}^{+}=0 & \text { on } \Gamma .\end{cases}
$$


Then the profile $\mathfrak{A}_{1}$ satisfies

$$
\mathfrak{A}_{1}(\xi, \Upsilon)=\left(\mathcal{A}_{1}^{+}(\mathbf{X}(\xi))+\frac{k(\xi)}{2} \mathcal{A}_{0}^{+}(\mathbf{X}(\xi)) \Upsilon\right) \mathrm{e}^{-\lambda_{0} \Upsilon}
$$

The next term $\mathcal{A}_{2}^{+}$solves the problem

$$
\begin{cases}\Delta \mathcal{A}_{2}^{+}=0 & \text { in } \Omega_{+} \\ \partial_{\mathbf{n}} \mathcal{A}_{2}^{+}=\frac{k(\xi)}{2} \mathcal{A}_{0}^{+}-\lambda_{0} \mathcal{A}_{1}^{+} & \text {on } \Sigma \\ \mathcal{A}_{2}^{+}=0 & \text { on } \Gamma\end{cases}
$$

Then the profile $\mathfrak{A}_{2}$ satisfies

$$
\mathfrak{A}_{2}(\xi, \Upsilon)=\left(\mathcal{A}_{2}^{+}+\left(\frac{k(\xi)}{2} \mathcal{A}_{1}^{+}+\frac{1}{2 \lambda_{0}}\left\{\partial_{\xi}^{2}+\frac{k(\xi)^{2}}{4} \mathbb{I}\right\} \mathcal{A}_{0}^{+}\right) \Upsilon+\frac{3 k(\xi)^{2}}{8} \mathcal{A}_{0}^{+} \Upsilon^{2}\right)(\mathbf{X}(\xi)) \mathrm{e}^{-\lambda_{0} \Upsilon}
$$

The next term which is determined is $\mathcal{A}_{3}^{+}$

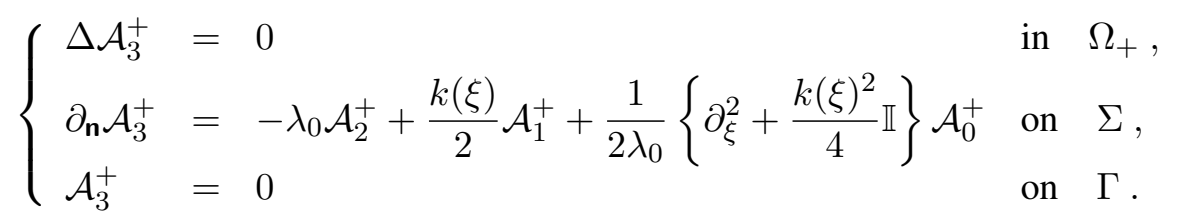

\section{EXISTENCE AND REGULARITY OF THE ASYMPTOTICS}

5.1. Regularity results for the first terms. It is possible to prove that Problem (4.1) (resp. (4.3), (4.5), (4.7)) has a unique weak solution $\mathcal{A}_{0}^{+}$(resp. $\left.\mathcal{A}_{l}^{+}, l=1,2,3\right)$ in the space $\mathrm{H}_{0, \Gamma}^{1}\left(\Omega_{+}\right)$ (Not. 2.2) when the right-hand side $J$ belongs to $L^{2}$ and the domain $\Omega_{+}$is sufficiently smooth. We first describe briefly the derivate consuming process for the first terms of the expansion (2.7).

The derivate consuming process of the expansion. We assume that $J \in \mathrm{L}^{2}\left(\Omega_{+}\right)$. Then, the problem (4.1) is solvable in the space $\mathrm{H}_{0, \Gamma}^{1}\left(\Omega_{+}\right)$. Since this problem is elliptic, the solution $\mathcal{A}_{0}^{+}$ has an optimal regularity which depends on the regularity of the data. Hence, $\mathcal{A}_{0}^{+} \in \mathrm{H}^{2}\left(\Omega_{+}\right) \cap$ $\mathrm{H}_{0, \Gamma}^{1}\left(\Omega_{+}\right)$and this solution satisfies an a priori estimate:

$$
\left\|\mathcal{A}_{0}^{+}\right\|_{2, \Omega_{+}} \leqslant C\|J\|_{0, \Omega_{+}}
$$

with a constant $C>0$ independent of $J$. Then, the trace $\left.\mathcal{A}_{0}^{+}\right|_{\Sigma}$ has to be inserted as a Neumann data into problem (4.3) defining $\mathcal{A}_{1}^{+}$. Since $\left.\mathcal{A}_{0}^{+}\right|_{\Sigma}$ belongs to $\mathrm{H}^{3 / 2}(\Sigma)$, the problem (4.3) is solvable in the space $\mathrm{H}_{0, \Gamma}^{1}\left(\Omega_{+}\right)$and the solution $\mathcal{A}_{1}^{+}$belongs to the Sobolev space $\mathrm{H}^{3}\left(\Omega_{+}\right)$. Furthermore, the solution satisfies the estimate:

$$
\left\|\mathcal{A}_{1}^{+}\right\|_{3, \Omega_{+}} \leqslant C\left\|\mathcal{A}_{0}^{+}\right\|_{3 / 2, \Sigma}
$$

and, from (5.1), we infer the estimate:

$$
\left\|\mathcal{A}_{1}^{+}\right\|_{3, \Omega_{+}} \leqslant C_{1}\|J\|_{0, \Omega_{+}} .
$$

Hence we win one rank of regularity to derive $\mathcal{A}_{1}^{+}$from the first asymptotic $\mathcal{A}_{0}^{+}$. Since $\left.\mathcal{A}_{0}^{+}\right|_{\Sigma} \in$ $\mathrm{H}^{3 / 2}(\Sigma)$ and $\left.\mathcal{A}_{1}^{+}\right|_{\Sigma} \in \mathrm{H}^{5 / 2}(\Sigma)$, the right-hand side on $\Sigma$ in Problem (4.5) belongs to $\mathrm{H}^{3 / 2}(\Sigma)$. 
Hence, the problem (4.5) is also solvable in $\mathrm{H}_{0, \Gamma}^{1}\left(\Omega_{+}\right)$and its solution $\mathcal{A}_{2}^{+}$belongs also to the Sobolev space $\mathrm{H}^{3}\left(\Omega_{+}\right)$. Furthermore, the solution satisfies the estimate:

$$
\left\|\mathcal{A}_{2}^{+}\right\|_{3, \Omega_{+}} \leqslant C\left(\left\|\mathcal{A}_{0}^{+}\right\|_{3 / 2, \Sigma}+\left\|\mathcal{A}_{1}^{+}\right\|_{5 / 2, \Sigma}\right) \leqslant C_{2}\|J\|_{0, \Omega_{+}} .
$$

Finally, the problem (4.7) has a unique solution $\mathcal{A}_{3}^{+}$in the space $\mathrm{H}_{0, \Gamma}^{1}\left(\Omega_{+}\right)$since the righthand side on $\Sigma$ belongs to the Sobolev space $\mathrm{H}^{-1 / 2}(\Sigma)$. Furthermore, the solution satisfies the estimate:

$$
\left\|\mathcal{A}_{3}^{+}\right\|_{1, \Omega_{+}} \leqslant C_{3}\|J\|_{0, \Omega_{+}} .
$$

Using general elliptic regularity result in Sobolev spaces for the Laplace operator, we prove regularity results in Sobolev spaces $\mathrm{H}^{s}$ for the first terms $\mathcal{A}_{l}^{+}$and $\mathfrak{A}_{l}, l=0,1,2,3$

Proposition 5.1. Let $k$ be an integer such that $k \geqslant 1$. We assume that the domain $\Omega_{+}$is smooth (of class $\mathcal{C}^{\infty}$ ). If J belongs to $\mathrm{H}^{k}\left(\Omega_{+}\right)$then

$$
\begin{gathered}
\mathcal{A}_{0}^{+} \in \mathrm{H}^{k+2}\left(\Omega_{+}\right) \quad \text { and } \mathfrak{A}_{0} \in \mathrm{H}^{k+\frac{3}{2}}\left(\Sigma \times \mathbb{R}^{+}\right), \\
\mathcal{A}_{1}^{+} \in \mathrm{H}^{k+3}\left(\Omega_{+}\right) \quad \text { and } \quad \mathfrak{A}_{1} \in \mathrm{H}^{k+\frac{3}{2}}\left(\Sigma \times \mathbb{R}^{+}\right), \\
\mathcal{A}_{2}^{+} \in \mathrm{H}^{k+3}\left(\Omega_{+}\right) \quad \text { and } \quad \mathfrak{A}_{2} \in \mathrm{H}^{k-\frac{1}{2}}\left(\Sigma \times \mathbb{R}^{+}\right), \\
\mathcal{A}_{3}^{+} \in \mathrm{H}^{k+1}\left(\Omega_{+}\right) .
\end{gathered}
$$

It is also possible to prove regularity results in analytic classes. We assume that $\Omega_{+}$is analytic. If $J$ is an analytic function on $\Omega_{+}$then each term $\mathcal{A}_{l}^{+}$is an analytic function on $\Omega_{+}$, see for instance [5, Th 3.4.2].

5.2. Synthesis of the multiscale expansion. Following the process of construction described above it is possible to derive the asymptotics $\mathcal{A}_{j}^{ \pm}$at any order when the right-hand side $J$ is of class $\mathcal{C}^{\infty}$. In this section we specify this result in Prop. 5.2.

The following definition for the resolvent operator $\mathcal{R}$ makes sense and define a bounded operator

$$
\begin{array}{ccc}
\mathcal{R}: \mathrm{H}^{\frac{1}{2}}(\Sigma) & \rightarrow & \left\{\varphi \in \mathrm{H}^{2}\left(\Omega_{+}\right) \mid \varphi=0 \quad \text { on } \Gamma\right\} \\
h & \mapsto & \varphi
\end{array}
$$

where $\varphi$ is the unique solution (in distributional sense) of the boundary value problem

$$
\begin{cases}\Delta \varphi=0 & \text { in } \Omega_{+}, \\ \partial_{\mathbf{n}}=h & \text { on } \Sigma, \\ \varphi=0 & \text { on } \Gamma .\end{cases}
$$

For all $t \geqslant 2$, the resolvent operator $\mathcal{R}$ is still a bounded operator in the following spaces

$$
\mathrm{H}^{t-\frac{3}{2}}(\Sigma) \rightarrow\left\{\varphi \in \mathrm{H}^{t}\left(\Omega_{+}\right) \mid \varphi=0 \quad \text { on } \Gamma\right\}
$$

Proposition 5.2. Let $s \geqslant 2$ and assume that $J \in \mathrm{H}^{s-2}(\Omega)$ has a support in $\Omega_{+}$. Assume that the interface $\Sigma$ is a curve of class $\mathcal{C}^{\infty}$. For all $j=0, \cdots,\lfloor s\rfloor+1$, it is possible to derive successively the following terms which are independent of $\varepsilon$

$$
\mathcal{A}_{j}^{+} \in \mathrm{H}^{s-j}\left(\Omega_{+}\right) \quad \text { and } \quad \mathfrak{A}_{j} \in \mathrm{H}^{s-j-\frac{3}{2}}\left(\Sigma, \mathcal{C}^{\infty}(I)\right) .
$$


For $j=0, \mathcal{A}_{0}^{+} \in \mathrm{H}^{s}\left(\Omega_{+}\right) \cap \mathrm{H}_{0, \Gamma}^{1}\left(\Omega_{+}\right)$is the solution of problem (4.1), and $\mathfrak{A}_{0}(\xi, \Upsilon)=$ $\mathcal{A}_{0}^{+}(\mathbf{X}(\xi)) \mathrm{e}^{-\lambda_{0} \Upsilon}$. For all $j=1, \cdots,\lfloor s\rfloor+1$, there exists a tangential operator $\mathfrak{T}_{j}$ which is defined as follow

$$
\begin{aligned}
\mathfrak{T}_{j}: \mathrm{H}^{s}\left(\Omega_{+}\right) & \rightarrow & \mathrm{H}^{s-j-\frac{3}{2}}(\Sigma) \\
\mathcal{A}_{0}^{+} & \mapsto & \left.\partial_{\mathbf{n}} \mathcal{A}_{j}^{+}\right|_{\Sigma}
\end{aligned}
$$

such that

$$
\mathcal{A}_{j}^{+}=\mathcal{R} \circ \mathfrak{T}_{j}\left(\mathcal{A}_{0}^{+}\right) .
$$

In particular for $j=1,2,3$, the operator $\mathfrak{T}_{j}$ is defined as

$$
\begin{aligned}
& \mathfrak{T}_{1}(\mathcal{A})=-\left.\lambda_{0} \mathcal{A}\right|_{\Sigma} \\
& \mathfrak{T}_{2}=\left(\mathfrak{T}_{1} \circ \mathcal{R}-\frac{k(\xi)}{2 \lambda_{0}} \mathbb{I}\right) \circ \mathfrak{T}_{1} \\
& \mathfrak{T}_{3}=\mathfrak{T}_{1} \circ \mathcal{R} \circ\left(\mathfrak{T}_{2}-\frac{k(\xi)}{2 \lambda_{0}} \mathfrak{T}_{1}\right)-\frac{1}{2 \lambda_{0}^{2}}\left(\partial_{\xi}^{2}+\frac{k(\xi)^{2}}{4} \mathbb{I}\right) \circ \mathfrak{T}_{1}
\end{aligned}
$$

Furthermore $\mathfrak{A}_{1}$ (resp. $\mathfrak{A}_{2}$ ) is defined by (4.4) (resp. (4.6)) and for all $n \geqslant 3$, the unique solution of the ODE (6.10) (see Sec. 6.2) such that $\mathfrak{A}_{n}(\cdot, \Upsilon) \longrightarrow 0$ as $\Upsilon \rightarrow \infty$ writes

$$
\mathfrak{A}_{n}(\xi, \Upsilon)=\left(a_{0}(\xi)+\Upsilon a_{1}(\xi)+\Upsilon^{2} a_{2}(\xi)+\cdots+\Upsilon^{n} a_{n}(\xi)\right) \mathrm{e}^{-\lambda_{0} \Upsilon}
$$

The solution $\mathcal{A}_{\varepsilon}$ of Problem (2.3) possesses the following asymptotic expansion

$$
\begin{gathered}
\mathcal{A}_{\varepsilon}^{+}(\mathbf{x})=\mathcal{A}_{0}^{+}(\mathbf{x})+\varepsilon \mathcal{A}_{1}^{+}(\mathbf{x})+\varepsilon^{2} \mathcal{A}_{2}^{+}(\mathbf{x})+\varepsilon^{3} \mathcal{A}_{3}^{+}(\mathbf{x})+\cdots \quad \text { in } \Omega_{+}, \\
\mathcal{A}_{\varepsilon}^{-}(\mathbf{x})=\chi(\nu) \mathfrak{A}_{0}\left(\xi, \frac{\nu}{\varepsilon}\right)+\varepsilon \chi(\nu) \mathfrak{A}_{1}\left(\xi, \frac{\nu}{\varepsilon}\right)+\varepsilon^{2} \chi(\nu) \mathfrak{A}_{2}\left(\xi, \frac{\nu}{\varepsilon}\right)++\cdots \quad \text { in } \Omega_{-},
\end{gathered}
$$

(2.7)-(2.8), see Theorem 2.4 for precise estimates.

\section{ELEMENTS OF DERIVATIONS FOR THE MULTISCALE EXPANSION AND IMPEDANCE CONDITIONS}

We assume that $\Sigma$ is a smooth $\left(\mathcal{C}^{\infty}\right)$ curve of length $L$. For $v_{0}>0$ small enough, the tubular neighborhood $\mathcal{V}(\Sigma)$ of $\Sigma$ of diameter $v_{0}$ in the inner domain $\Omega_{-}$is parameterized by

$$
\mathcal{V}(\Sigma)=\left\{\mathbf{x}(\xi, v)=\mathbf{X}(\xi)+v \mathbf{n}(\xi), \quad(\xi, v) \in \Sigma \times\left(0, v_{0}\right)\right\},
$$

See Notation 4.1. The system of coordinates $(\xi, v)$ is orthogonal, and the first fundamental form writes

$$
Q^{2}(\xi, v)(d \xi)^{2}+(d v)^{2}, \quad \text { with } \quad Q(\xi, v)=(1-v k(\xi)),
$$

where $k(\xi)$ denotes the curvature at the point $\mathbf{X}(\xi)$ (Notation 4.1). For $v_{0}<1 /\|k\|_{\infty}$ the change of coordinates

$$
\Psi: \mathbf{x}=(x, y) \longmapsto(\xi, v)
$$

is a $\mathcal{C}^{\infty}$-diffeomorphism from $\mathcal{V}(\Sigma)$ into the cylinder $\mathbb{T}_{L} \times\left(0, v_{0}\right)$ :

$$
\mathcal{V}(\Sigma)=\Psi^{-1}\left(\mathbb{T}_{L} \times\left(0, v_{0}\right)\right) .
$$

Hereafter, in Sec. 6.2 we set the ansatz (2.7)-(2.8)-(2.9) for the solution of problem (2.3). The function $(\xi, v) \mapsto \chi(v)$ which appear in (2.9) is a smooth cut-off function whose support is included in $\overline{\mathcal{V}(\Sigma)}$, and equal to 1 in a smaller tubular neighborhood of the interface. 
6.1. Expansion of the operators in power series of $\varepsilon$. In order to exhibit the formal expansion of $\mathcal{A}_{\varepsilon}^{-}$we first use the change of variables $\Psi$ in order to write the equations in the cylinder $\mathbb{T}_{L} \times\left(0, v_{0}\right)$. We then perform the rescaling

$$
\Upsilon=\frac{v}{\varepsilon}
$$

in the equations set in $\Omega_{-}$and $\Sigma$ in order to make appear the small parameter $\varepsilon$ in the equations. The Laplace operator writes in coordinates $(\xi, \nu)$ as

$$
\Delta=Q^{-1}\left[\varepsilon^{-2} \partial_{\Upsilon}\left(Q \partial_{\Upsilon}\right)+\partial_{\xi}\left(Q^{-1} \partial_{\xi}\right)\right]
$$

where $Q=(1-\varepsilon \Upsilon k(\xi))$. This operator $\Delta$ (6.3) expands in power series of $\varepsilon$ with coefficients intrinsic operators with respect to $\Sigma$

$$
\Delta=\frac{1}{\varepsilon^{2}} \partial_{\Upsilon}^{2}+\sum_{n \geqslant-1} \varepsilon^{n} D_{n} .
$$

Note that this expansion (6.4) is a convergent power series expansion when $\Upsilon \in I_{\varepsilon}=\left(0, \frac{\nu_{0}}{\varepsilon}\right)$. Here for all $n \geq-1, D_{n}=D_{n}\left(\xi, \Upsilon ; \partial_{\xi}, \partial_{\Upsilon}\right)$ is an operator with at most one derivative in the $\Upsilon$-variable, which has smooth coefficients in $\xi$, and polynomial coefficients of degree $n-1$ in $\Upsilon$. Note that in particular $D_{-1}=-k(\xi) \partial_{\Upsilon}$. In the following we shall use the operator $\mathrm{A}_{n}:=D_{n-2}$

$$
\varepsilon^{2} \Delta=\partial_{\Upsilon}^{2}+\sum_{n \geqslant 1} \varepsilon^{n} \mathrm{~A}_{n} .
$$

Observe that

$$
\begin{gathered}
\mathrm{A}_{1}=-k(\xi) \partial_{\Upsilon} \\
\mathrm{A}_{2}=\partial_{\xi}^{2}-k(\xi)^{2} \Upsilon \partial_{\Upsilon}, \\
\mathrm{A}_{3}=-k(\xi)^{3} \Upsilon^{2} \partial_{\Upsilon}+\Upsilon k^{\prime}(\xi) \partial_{\xi}+2 \Upsilon k(\xi) \partial_{\xi}^{2}
\end{gathered}
$$

Similarly we write

on the interface $\Sigma$.

$$
\partial_{\mathbf{n}}\left(\xi ; \partial_{\Upsilon}\right)=\frac{1}{\varepsilon} \partial_{\Upsilon}
$$

6.2. Equations for the coefficients of the magnetic potential. In this section, define $\mathrm{v}_{\varepsilon}$ by $\mathrm{v}_{\varepsilon}(\xi, \Upsilon)=\mathcal{A}_{\varepsilon}^{-}(\mathbf{x})$ in $\mathcal{V}(\Sigma)$. After the scaling $v \mapsto \Upsilon=\frac{v}{\varepsilon}$ in $\mathcal{V}(\Sigma)$, the problem (2.3) writes

$$
\begin{cases}-\Delta \mathcal{A}_{\varepsilon}^{+}=\mu_{+} J & \text { in } \quad \Omega_{+} \\ \partial_{\mathbf{n}} \mathcal{A}_{\varepsilon}^{+}=\varepsilon \partial_{\Upsilon v_{\varepsilon}} & \text { on } \Sigma \\ \mathcal{A}_{\varepsilon}^{+}=0 & \text { on } \Gamma\end{cases}
$$

and

$$
\left\{\begin{array}{lll}
\left(-\partial_{\Upsilon}^{2}+\frac{\mathrm{i}}{\delta_{0}^{2}}\right) \mathrm{v}_{\varepsilon}-\sum_{n \geqslant 1} \varepsilon^{n} \mathrm{~A}_{n} \mathrm{v}_{\varepsilon} & =0 & \text { in } \quad \mathbb{T}_{L} \times(0,+\infty), \\
\mathrm{v}_{\varepsilon} & =\mathcal{A}_{\varepsilon}^{+} & \text {on } \quad \mathbb{T}_{L} \times\{0\}
\end{array}\right.
$$

We insert the Ansatz

$$
\mathcal{A}_{\varepsilon}^{+} \sim \sum_{n \geqslant 0} \varepsilon^{n} \mathcal{A}_{n}^{+}(\mathbf{x}) \quad \text { in } \quad \Omega_{+}, \quad \text { and } \quad \mathrm{v}_{\varepsilon} \sim \sum_{n \geqslant 0} \varepsilon^{n} \mathfrak{A}_{n}(\xi, \Upsilon) \quad \text { in } \quad \mathcal{V}(\Sigma)
$$


with $\mathfrak{A}_{n}(\cdot, \Upsilon) \longrightarrow 0$ as $\Upsilon \rightarrow \infty$, in equations (6.6) and (6.7), and we perform the identification of terms with the same power in $\varepsilon$. Setting

$$
\lambda_{0}=\frac{1}{\delta_{0}} \frac{\sqrt{2}}{2}(1+\mathrm{i})
$$

in equation (6.7), then the terms $\mathfrak{A}_{n}$ and $\mathcal{A}_{n}^{+}$satisfy the following family of problems coupled by their conditions on the interface $\Sigma$ (corresponding to $\Upsilon=0$ ):

$$
\left\{\begin{array}{lll}
-\Delta \mathcal{A}_{n}^{+} & =\mu_{+} J \delta_{n}^{0} & \text { in } \quad \Omega_{+}, \\
\partial_{\mathbf{n}} \mathcal{A}_{n}^{+} & =\partial_{\Upsilon} \mathfrak{A}_{n-1} & \text { on } \quad \Sigma, \\
\mathcal{A}_{n}^{+} & =0 & \text { on } \quad \Gamma
\end{array}\right.
$$

and

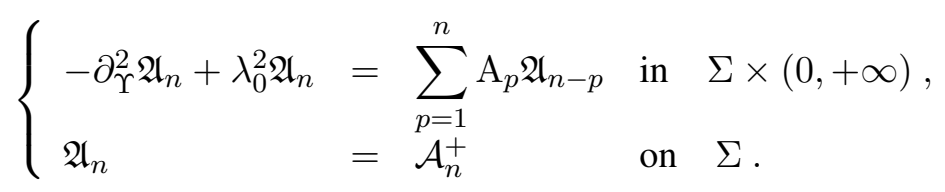

In (6.9) $\delta_{n}^{0}$ denotes the Kronecker symbol and we use the convention $\mathfrak{A}_{-1}=0$. Hereafter, we make explicit the first asymptotics $\mathfrak{A}_{n}$ and $\mathcal{A}_{n}^{+}$for $n=0,1,2$ by induction.

6.3. First terms of the asymptotics for the magnetic potential. The first terms which are determined are $\mathcal{A}_{0}^{+}$and $\mathfrak{A}_{0}$. We obtain from (6.9) that $\mathcal{A}_{0}^{+}$solves the problem

$$
\left\{\begin{array}{lll}
-\Delta \mathcal{A}_{0}^{+}=\mu_{+} J & \text { in } \Omega_{+}, \\
\partial_{\mathbf{n}} \mathcal{A}_{0}^{+}=0 & \text { on } \Sigma, \\
\mathcal{A}_{0}^{+}=0 & \text { on } \Gamma .
\end{array}\right.
$$

Then according to (6.10) in the case $n=0, \mathfrak{A}_{0}$ solves the ODE

$$
\left\{\begin{array}{lll}
-\partial_{\Upsilon}^{2} \mathfrak{A}_{0}+\lambda_{0}^{2} \mathfrak{A}_{0} & =0 & \text { in } \Sigma \times(0,+\infty), \\
\mathfrak{A}_{0} & =\mathcal{A}_{0}^{+} \text {on } \Sigma .
\end{array}\right.
$$

The unique solution of (6.12) such that $\mathfrak{A}_{0} \longrightarrow 0$ when $\Upsilon \rightarrow \infty$ is

$$
\mathfrak{A}_{0}(\xi, \Upsilon)=\mathcal{A}_{0}^{+}(\mathbf{X}(\xi)) \mathrm{e}^{-\lambda_{0} \Upsilon} .
$$

The next term which is determined is $\mathcal{A}_{1}^{+}$. According to (6.9) in the case $n=1$ and according to (6.13), $\mathcal{A}_{1}^{+}$solves the following problem

$$
\begin{cases}\Delta \mathcal{A}_{1}^{+}=0 & \text { in } \Omega_{+}, \\ \partial_{\mathbf{n}} \mathcal{A}_{1}^{+}=\partial_{\Upsilon} \mathfrak{A}_{0}=-\lambda_{0} \mathcal{A}_{0}^{+} & \text {on } \Sigma, \\ \mathcal{A}_{1}^{+}=0 & \text { on } \Gamma .\end{cases}
$$

Then according to (6.10) for $n=1, \mathfrak{A}_{1}$ solves the ODE

$$
\begin{cases}-\partial_{\Upsilon}^{2} \mathfrak{A}_{1}(\cdot, \Upsilon)+\lambda_{0}^{2} \mathfrak{A}_{1}(\cdot, \Upsilon) & =\lambda_{0} k(\xi) \mathcal{A}_{0}^{+}(\mathbf{X}(\xi)) \mathrm{e}^{-\lambda_{0} \Upsilon} \text { for } \Upsilon \in(0,+\infty) \\ \mathfrak{A}_{1}(\cdot, 0) & =\mathcal{A}_{1}^{+}(\mathbf{X}(\xi))\end{cases}
$$


The unique solution of (6.15) such that $\mathfrak{A}_{1} \rightarrow 0$ when $\Upsilon \rightarrow \infty$ is

$$
\mathfrak{A}_{1}(\xi, \Upsilon)=\left(\mathcal{A}_{1}^{+}(\mathbf{X}(\xi))+\frac{k(\xi)}{2} \mathcal{A}_{0}^{+}(\mathbf{X}(\xi)) \Upsilon\right) \mathrm{e}^{-\lambda_{0} \Upsilon}
$$

The next term which is determined is $\mathcal{A}_{2}^{+}$. According to (6.9) in the case $n=2$ and according to (6.16), $\mathcal{A}_{2}^{+}$solves the following problem

$$
\left\{\begin{array}{lll}
\Delta \mathcal{A}_{2}^{+} & =0 & \text { in } \quad \Omega_{+} \\
\partial_{\mathbf{n}} \mathcal{A}_{2}^{+} & =\partial_{\Upsilon} \mathfrak{A}_{1}=\frac{k(\xi)}{2} \mathcal{A}_{0}^{+}-\lambda_{0} \mathcal{A}_{1}^{+} & \text {on } \Sigma \\
\mathcal{A}_{2}^{+} & =0 & \text { on } \quad \Gamma
\end{array}\right.
$$

According to (6.10) for $n=2, \mathfrak{A}_{2}$ solves the ODE

$$
\begin{cases}-\partial_{\Upsilon}^{2} \mathfrak{A}_{2}(\cdot, \Upsilon)+\lambda_{0}^{2} \mathfrak{A}_{2}(\cdot, \Upsilon) & =-k(\xi) \partial_{\Upsilon} \mathfrak{A}_{1}+\mathrm{A}_{2} \mathfrak{A}_{0} \text { for } \Upsilon \in(0,+\infty) \\ \mathfrak{A}_{2}(\cdot, 0) & =\mathcal{A}_{2}^{+}(\mathbf{X}(\xi))\end{cases}
$$

We can explicit the right-hand side of this ODE. According to (6.16)

$$
\partial_{\Upsilon} \mathfrak{A}_{1}=\left(\frac{k(\xi)}{2} \mathcal{A}_{0}^{+}-\lambda_{0}\left(\mathcal{A}_{1}^{+}+\frac{k(\xi)}{2} \mathcal{A}_{0}^{+} \Upsilon\right)\right) \mathrm{e}^{-\lambda_{0} \Upsilon}
$$

and according to (6.5b) and (6.13) we infer successively

$$
\mathrm{A}_{2} \mathfrak{A}_{0}(\xi, \Upsilon)=\left\{\partial_{\xi}^{2}-k(\xi)^{2} \Upsilon \partial_{\Upsilon}\right\} \mathfrak{A}_{0}(\xi, \Upsilon)=\left(\partial_{\xi}^{2} \mathcal{A}_{0}^{+}+\lambda_{0} k(\xi)^{2} \Upsilon \mathcal{A}_{0}^{+}\right)(\mathbf{X}(\xi)) \mathrm{e}^{-\lambda_{0} \Upsilon} .
$$

Then the unique solution of the ODE (6.18) such that $\mathfrak{A}_{2} \rightarrow 0$ when $\Upsilon \rightarrow \infty$ is

$$
\mathfrak{A}_{2}(\xi, \Upsilon)=\left(\mathcal{A}_{2}^{+}+\left(\frac{k(\xi)}{2} \mathcal{A}_{1}^{+}+\frac{1}{2 \lambda_{0}}\left\{\partial_{\xi}^{2}+\frac{k(\xi)^{2}}{4} \mathbb{I}\right\} \mathcal{A}_{0}^{+}\right) \Upsilon+\frac{3 k(\xi)^{2}}{8} \mathcal{A}_{0}^{+} \Upsilon^{2}\right)(\mathbf{X}(\xi)) \mathrm{e}^{-\lambda_{0} \Upsilon}
$$

The next term which is determined is $\mathcal{A}_{3}^{+}$. According to (6.9) in the case $n=3$ and according to (6.19), $\mathcal{A}_{3}^{+}$solves the following problem

$$
\begin{cases}\Delta \mathcal{A}_{3}^{+}=0 \\
\partial_{\mathbf{n}} \mathcal{A}_{3}^{+}=\partial_{\Upsilon} \mathfrak{A}_{2}=-\lambda_{0} \mathcal{A}_{2}^{+}+\frac{k(\xi)}{2} \mathcal{A}_{1}^{+}+\frac{1}{2 \lambda_{0}}\left\{\partial_{\xi}^{2}+\frac{k(\xi)^{2}}{4} \mathbb{I}\right\} \mathcal{A}_{0}^{+} & \text {in } \quad \begin{array}{l}
\Omega_{+} \\
\mathcal{A}_{2}^{+}=0
\end{array} \\
\text { on } \quad \Gamma\end{cases}
$$

6.4. Construction of impedance conditions. In this section, we derive high-order impedance boundary conditions (see Sec. 2.3.1) set on the interface $\Sigma$ for the solution of the problem (2.3).

Order 1 . Since $\mathcal{A}_{0}^{+}$solves the limit problem (4.1), the condition of order 1 is the perfect magnetic conductor boundary condition, see (2.12)

$$
\partial_{\mathbf{n}} \mathcal{A}_{0}=0 \quad \text { on } \Sigma .
$$


Order 2. According to (4.1)-(4.3), the truncated expansion $\mathcal{A}_{1, \varepsilon}^{+}:=\mathcal{A}_{0}^{+}+\varepsilon \mathcal{A}_{1}^{+}$solves the Laplace equation $-\Delta \mathcal{A}_{1, \varepsilon}^{+}=\mu_{+} J$ in $\Omega_{+}$together with the boundary condition set on $\Sigma$

$$
\partial_{\mathbf{n}} \mathcal{A}_{1, \varepsilon}^{+}+\lambda_{0} \varepsilon \mathcal{A}_{1, \varepsilon}^{+}=\lambda_{0} \varepsilon^{2} \mathcal{A}_{1}^{+} \quad \text { on } \quad \Sigma .
$$

Neglecting the term of order 2 in the previous right-hand side, we infer the Leontovich boundary condition, see (2.13)

$$
\partial_{\mathbf{n}} \mathcal{A}_{1}^{\varepsilon}+\lambda_{0} \varepsilon \mathcal{A}_{1}^{\varepsilon}=0 \quad \text { on } \quad \Sigma .
$$

Order 3. According to (4.1)-(4.3)-(4.5), the truncated expansion $\mathcal{A}_{2, \varepsilon}^{+}:=\mathcal{A}_{0}^{+}+\varepsilon \mathcal{A}_{1}^{+}+\varepsilon^{2} \mathcal{A}_{2}^{+}$ solves the Laplace equation $-\Delta \mathcal{A}_{2, \varepsilon}^{+}=\mu_{+} J$ in $\Omega_{+}$together with the boundary condition set on $\Sigma$

$$
\partial_{\mathbf{n}} \mathcal{A}_{2, \varepsilon}^{+}+\varepsilon\left(\lambda_{0}-\frac{k(\xi)}{2} \varepsilon\right) \mathcal{A}_{2, \varepsilon}^{+}=\varepsilon^{3}\left(-\frac{k(\xi)}{2} \mathcal{A}_{1}^{+}+\left(\lambda_{0}-\frac{k(\xi)}{2} \varepsilon\right) \mathcal{A}_{2}^{+}\right) \quad \text { on } \quad \Sigma
$$

We neglect the terms in $\mathcal{O}\left(\varepsilon^{3}\right)$ in the previous right-hand side. Then we infer the impedance boundary condition of order 3 , see (2.14)

$$
\partial_{\mathbf{n}} \mathcal{A}_{2}^{\varepsilon}+\varepsilon\left(\lambda_{0}-\frac{k(\xi)}{2} \varepsilon\right) \mathcal{A}_{2}^{\varepsilon}=0 \quad \text { on } \quad \Sigma .
$$

Order 4 . We identify a simpler problem satisfied by the truncated expansion $\mathcal{A}_{3, \varepsilon}^{+}:=\mathcal{A}_{0}^{+}+$ $\varepsilon \mathcal{A}_{1}^{+}+\varepsilon^{2} \mathcal{A}_{2}^{+}+\varepsilon^{3} \mathcal{A}_{3}^{+}$

$$
\left\{\begin{array}{lll}
-\Delta \mathcal{A}_{3, \varepsilon}^{+} & =\mu_{+} J & \text { in } \Omega_{+}, \\
\partial_{\mathbf{n}} \mathcal{A}_{3, \varepsilon}^{+}+\mathrm{N}_{3, \varepsilon}\left(\mathcal{A}_{3, \varepsilon}^{+}\right) & =\mathcal{O}\left(\varepsilon^{4}\right) & \text { on } \Sigma, \\
\mathcal{A}_{3, \varepsilon}^{+} & =0 & \text { on } \Gamma,
\end{array}\right.
$$

where $\mathrm{N}_{3, \varepsilon}=\varepsilon\left(\left(\lambda_{0}-\frac{k(\xi)}{2} \varepsilon\right) \mathbb{I}-\frac{\varepsilon^{2}}{2 \lambda_{0}}\left\{\partial_{\xi}^{2}+\frac{k(\xi)^{2}}{4} \mathbb{I}\right\}\right)$. We neglect the terms in $\mathcal{O}\left(\varepsilon^{4}\right)$ in the right-hand sides. Then we infer the impedance boundary condition of order 4 , see (2.15).

\section{ACKNOWLEDGEMENTS}

The author V. P. thanks very warmly Monique Dauge for her well-considered suggestions. 


\section{REFERENCES}

[1] X. Antoine, H. Barucq, and L. Vernhet. High-frequency asymptotic analysis of a dissipative transmission problem resulting in generalized impedance boundary conditions. Asymptot. Anal., 26(3-4):257-283, 2001.

[2] A. Bendali and K. Lemrabet. The effect of a thin coating on the scattering of a time-harmonic wave for the Helmholtz equation. SIAM J. Appl. Math., 56(6):1664-1693, 1996.

[3] G. Caloz, M. Dauge, E. Faou, and V. Péron. On the influence of the geometry on skin effect in electromagnetism. Computer Methods in Applied Mechanics and Engineering, 200(9-12):1053-1068, 2011.

[4] J. Chabassier, M. Duruflé, and V. Péron. Equivalent boundary conditions for acoustic media with exponential densities. Application to the atmosphere in helioseismology. Applied Mathematics and Computation, 361:177197, November 2019.

[5] M. Costabel, M. Dauge, and S. Nicaise. Corner Singularities and Analytic Regularity for Linear Elliptic Systems. Part I: Smooth domains. 211 pages v2: Improvement of layout.

[6] M. Dauge, E. Faou, and V. Péron. Comportement asymptotique à haute conductivité de l'épaisseur de peau en électromagnétisme. C. R. Math. Acad. Sci. Paris, 348(7-8):385-390, 2010.

[7] B. Engquist and J.C. Nédélec. Effective boundary condition for acoustic and electromagnetic scattering in thin layers. Technical Report of CMAP, 278, 1993.

[8] H. Haddar, P. Joly, and H.-M. Nguyen. Generalized impedance boundary conditions for scattering by strongly absorbing obstacles: the scalar case. Math. Models Methods Appl. Sci., 15(8):1273-1300, 2005.

[9] N. Ida and S. Yuferev. Impedance boundary conditions for transient scattering problems. Magnetics, IEEE Transactions on, 33(2):1444-1447, 1997.

[10] L. Krähenbühl, V. Péron, R. Perrussel, and C. Poignard. On the asymptotic expansion of the magnetic potential in eddy current problem: a practical use of asymptotics for numerical purposes. Research Report RR-8749, INRIA Bordeaux, June 2015.

[11] O. D. Lafitte and G. Lebeau. Équations de Maxwell et opérateur d'impédance sur le bord d'un obstacle convexe absorbant. C. R. Acad. Sci. Paris Sér. I Math., 316(11):1177-1182, 1993.

[12] M. A. Leontovich. Approximate boundary conditions for the electromagnetic field on the surface of a good conductor. In Investigations on radiowave propagation, volume 2, pages 5-12. Printing House of the USSR Academy of Sciences, Moscow, 1948.

[13] R. C. MacCamy and E. Stephan. A skin effect approximation for eddy current problems. Arch. Rational Mech. Anal., 90(1):87-98, 1985.

[14] V. Péron. Asymptotic expansion for the magnetic potential in the eddy current problem : the ferromagnetic case. working paper or preprint, 2015.

[15] S. M. Rytov. Calcul du skin effect par la méthode des perturbations. Journal of Physics, 11(3):233-242, 1940.

[16] T.B.A. Senior, J.L. Volakis, and Institution of Electrical Engineers. Approximate Boundary Conditions in Electromagnetics. IEE Electromagnetic Waves Series. Inst of Engineering \& Technology, 1995.

(V. Péron) Université de Pau et des Pays de L'Adour, E2S UPPA, CNRS, INRIA, Équipe Magique 3D, LMAP CNRS UMR 5142, 64013 PAU, FRANCE

Email address, V. Péron: victor.peroneuniv-pau.fr

(C. Poignard) INRIA, Bordeaux INP, CNRS, Univ. Bordeaux, IMB, UMR 5251, F-33400 Talence, FRANCE.

Email address, C. Poignard: clair.poignardeinria.fr 